\title{
School level of children carrying a HNF1B variant or a deletion
}

\author{
Fanny Laliève ${ }^{1} \cdot$ Stéphane Decramer ${ }^{2}$ Laurence Heidet ${ }^{3} \cdot$ Véronique Baudouin $^{4} \cdot$ Annie Lahoche $^{5} \cdot$ Brigitte Llanas $^{6}$. \\ ${\text { Pierre } \text { Cochat }^{7} \text { - Julie Tenenbaum }}^{8}$ - Marie-Pierre Lavocat ${ }^{9}$. Philippe Eckart ${ }^{10}$. Françoise Broux ${ }^{11}$. \\ Gwenaelle Roussey $^{12} \cdot$ Sylvie Cloarec $^{13} \cdot$ Isabelle Vrillon $^{14} \cdot$ Olivier Dunand $^{15} \cdot$ Lucie Bessenay $^{16}$. \\ Michel Tsimaratos ${ }^{17}$. François Nobili ${ }^{18}$. Christine Pietrement ${ }^{19} \cdot$ Loïc De Parscau $^{20}$ • Valérie Bonneville ${ }^{21}$. \\ Nicolas Rodier $^{22}$ - Cécile Saint-Martin ${ }^{23}$ - Nicolas Chassaing ${ }^{24} \cdot$ Laurence Michel-Calemard $^{25}$ - Vincent Moriniere ${ }^{26}$. \\ Christine Bellanné-Chantelot ${ }^{23}$ - Claire Bahans ${ }^{1} \cdot$ Vincent Guigonis $\mathbb{C}^{1,22,27}$
}

Received: 18 December 2017 / Revised: 2 July 2019 / Accepted: 16 July 2019 / Published online: 3 September 2019

(c) The Author(s), under exclusive licence to European Society of Human Genetics 2019

\begin{abstract}
The prevalence of neurological involvement in patients with a deletion of or a variant in the HNF1B gene remains discussed. The aim of this study was to investigate the neuropsychological outcomes in a large cohort of children carrying either a $H N F 1 B$ whole-gene deletion or a disease-associated variant, revealed by the presence of kidney anomalies. The neuropsychological development-based on school level—of 223 children included in this prospective cohort was studied. Data from 180 children were available for analysis. Patients mean age was 9.6 years, with $39.9 \%$ of girls. Among these patients, 119 carried a $H N F 1 B$ deletion and 61 a disease-associated variant. In the school-aged population, 12.7 and $3.6 \%$ of patients carrying a $H N F 1 B$ deletion and a disease-associated variant had special educational needs, respectively. Therefore, the presence of a $H N F 1 B$ deletion increases the risk to present with a neuropsychiatric involvement when compared with the general population. On the other hand, almost $90 \%$ of patients carrying a $H N F 1 B$ disease-associated variant or deletion have a normal schooling in a general educational environment. Even if these findings do not predict the risk of neuropsychiatric disease at adulthood, most patients diagnosed secondary to kidney anomalies do not show a neurological outcome severe enough to impede standard schooling at elementary school. These results should be taken into account in prenatal counseling.
\end{abstract}

\section{Introduction}

Hepatocyte nuclear factor $1 \mathrm{~B}(H N F 1 B)$ is a gene coding a transcription factor expressed in polarized epithelia. It plays a role in the regulation of gene expression and the development of various tissues, such as kidney, pancreas, liver, bowel, and genital organs [1,2]. Clinically, patients may present with renal cysts, diabetes, hepatic cytolysis, and/or gynecological abnormalities [3]. Molecularly, $H N F 1 B$ anomalies may be secondary to a single-nucleotide variant (SNV) or a copy number variant $(\mathrm{CNV})$ resulting in a complete deletion of the gene, the latter being part of a recurrent chromosomal microdeletion (about $1.3 \mathrm{Mb}$,

Fanny Laliève

lalieve.fanny@gmail.com

Vincent Guigonis

vincent.guigonis@unilim.fr

Extended author information available on the last page of the article. encompassing 14 other genes), often referred to as $17 \mathrm{q} 12$ microdeletion diagnosed with cytogenetic diagnosis tools, such as CGH array and FISH. We have previously demonstrated that all tested patients with a HNF1B deletion carry a $17 \mathrm{q} 12$ deletion [4]. Therefore, all patients with a $H N F 1 B$ deletion should be considered as carrying a $17 \mathrm{q} 12$ deletion even when no CGH array or FISH has been performed.

The prevalence of neurological involvement in patients with HNF1B SNV or CNV (i.e., 17q12 microdeletion) remains discussed. Indeed, for some authors, the $17 \mathrm{q} 12$ deletion is associated with a high risk of neuropsychological disorders, such as schizophrenia, autism, and learning disabilities (when comparing a large sample of patients with neurodevelopmental disorders and controls [5], or in small cohort of patients carrying 17q12 deletion [6]), whereas a severe neuropsychological involvement has rarely been described in cohorts of patients with HNFIB SNV or CNV diagnosed secondary to renal diseases [3, 7]. In 2016, Clissold et al. have studied 38 patients with renal disease 
secondary to either a $H N F 1 B$ CNV $(n=20)$ or an SNV ( $n$ $=18$ ). Based on the evaluation criteria including severe and moderate disorders, they have reported up to $40 \%$ of neuropsychological disorders, but only in patients carrying the deletion [8]. More recently, Dubois-Laforgue et al. have reported a prevalence of intellectual and/or learning disabilities of $22.4 \%$ in a large cohort of 107 adult patients with HNF1B-related diabetes (MODY5) [9]. In this study, neuropsychological disorders were present in both groups of patients (carrying either an SNV or a CNV). Actually, the real prevalence of neuropsychological disorders in patients with a $17 \mathrm{q} 12$ microdeletion is not known. Indeed, MorenoDe-Luca et al. have described 17q12 deletions in patients selected because they had neuropsychological disorders $[5,6,10]$. Therefore, such an approach cannot be used to estimate the prevalence of neuropsychological disorders in the whole population of patients carrying a $17 \mathrm{q} 12$ microdeletion (or $H N F 1 B$ deletion). On the other hand, studies assessing cohorts of patients with renal diseases, other than the recent reports by Clissold and Dubois-Laforgue, could have underestimated the prevalence of neuropsychological disorders in patients carrying a HNF1B SNV or CNV for several reasons such as underdiagnosis of moderate neurological abnormalities, milder neurological disorders in patients with a $H N F 1 B$ disease-associated variant or initial patient selection bias.

Based on these data on the neurological outcome and considering that the diagnosis of HNFIB SNV or CNV is now frequently suspected prenatally based on kidney development anomalies (i.e., hyperechogenic fetal kidneys on prenatal ultrasound) [11], the information about the neurological outcome, especially the frequency of severe involvement, is crucial to provide reliable antenatal counseling to parents. Therefore, the neuropsychological phenotypes of pediatric patients carrying either a HNF1B SNV or $\mathrm{CNV}$ deserve to be clarified.

The aim of this study was to investigate the neuropsychological outcomes, based on the school level, in a large cohort of pediatric patients with renal anomalies carrying either a $H N F 1 B$ whole deletion or a disease-associated variant.

\section{Patients and methods}

\section{Patients}

Since 2008, within the French Society for Pediatric Nephrology (Société de Néphrologie Pédiatrique) network, a molecular analysis of HNF1B (based on a method described elsewhere [12]) is regularly proposed to children presenting with a compatible kidney phenotype. Children with confirmed $H N F 1 B$ abnormalities (either $\mathrm{CNV}$ or disease-associated SNV) are invited to participate in a prospective cohort study with a standardized follow-up. Included patients are evaluated annually (including physical examination, biological test, and renal ultrasound). Almost all Pediatric Nephrology units in France $(n=21)$ participate in this cohort study so that, although it is not exhaustive, the inclusion rate of diagnosed patients is high (about $50 \%$ of diagnosed cases based on the number of positive molecular diagnostic tests performed by the four referent diagnostic laboratories in France, data not shown).

The neurological data of these patients are presented in this study.

\section{Study design}

Since September 2013, the neuropsychological data of all patients included in the cohort were prospectively collected during the annual follow-up visit. The following items were collected using a standardized form: school level, learning difficulties, behavioral disorders, and neurological or psychiatric diagnoses if appropriate. Data from the last followup of each patient were analyzed in this study.

The primary objective was to assess the prevalence of neuropsychological impairments that were severe enough to disrupt schooling in patients carrying a HNFIB SNV or $\mathrm{CNV}$ diagnosed secondary to renal anomalies. A normal school level was defined as being in the class \pm 1 matching patient age. In France, due to an automatic grade promotion policy, children who fall outside this criterion have severe learning difficulties. Therefore, it was expected that children with a "abnormal" school level required special education. The secondary objective was to compare these results between patients with a $H N F 1 B$ SNV or CNV.

As molecular analyses of $H N F 1 B$ gene were performed in four different laboratories, genetic results of all the patients included were reviewed by two authors (CS-M and CB-C) in order to classify each SNV according to HGVS recommendations (i.e., affects or probably affects function) [13]. These data are available at http://www.lovd.nl/HNF1B (Patient IDs 231052-231259).

Data were collected, centralized, and analyzed in an electronic database.

This prospective study was reviewed and approved by an institutional ethic committee (CPP Sud-Ouest Outre Mer IV) and registered in the North American Clinical Trial database (NCT00760331). All parents signed an informed consent before inclusion of their children in this study.

\section{Statistics}

Baseline data are represented by means and standard deviations for continuous data, or by number and percentages for categorical data. The prevalence of neuropsychological 
Fig. 1 Flow chart

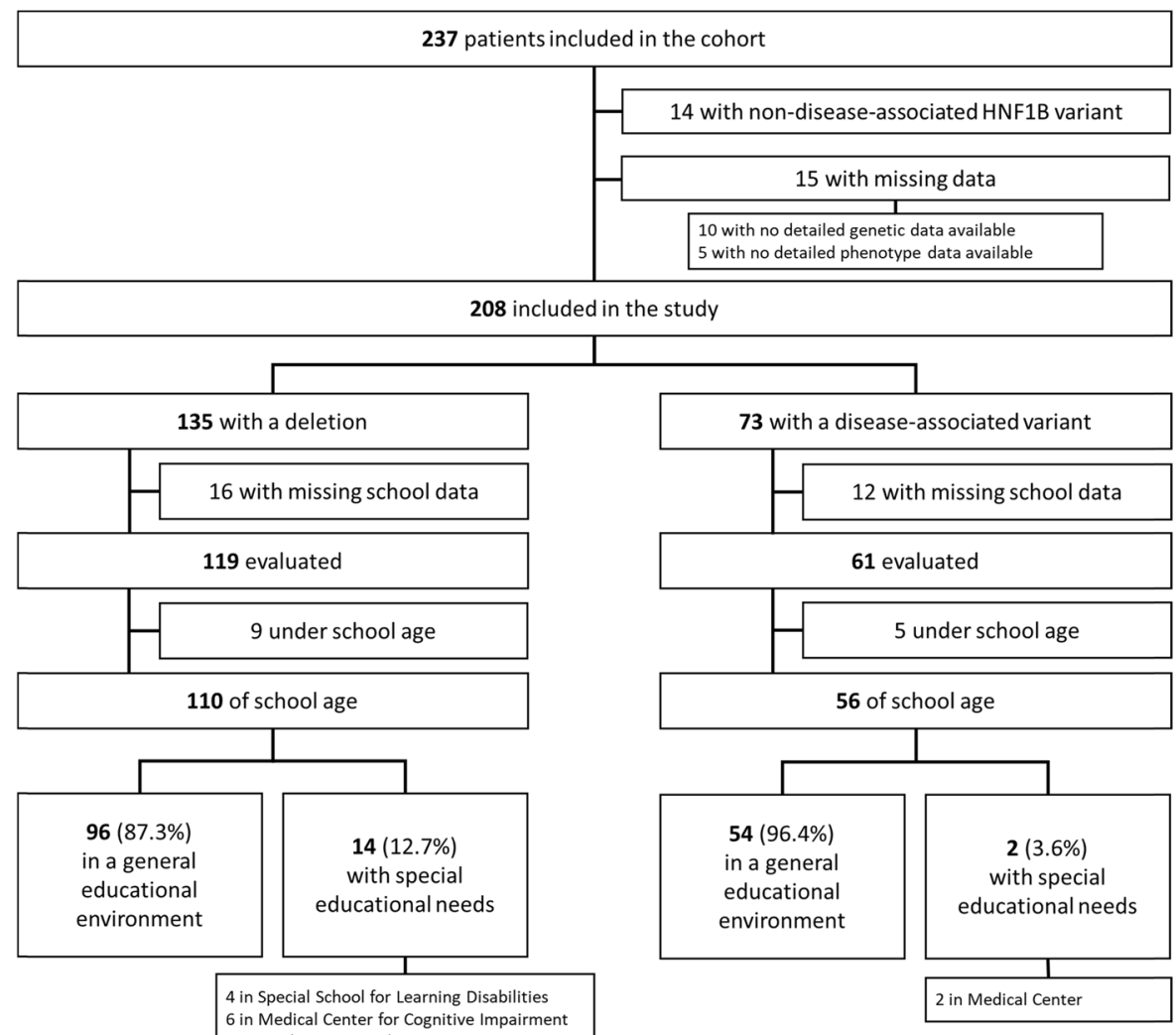

impairment in children with $H N F 1 B$ abnormalities is represented as a percentage with its confidence interval. Continuous data were compared using a Mann-Whitney test. Categorical variables were compared using a $\chi^{2}$ test or a Fisher's exact test (small sample size). Results were considered statistically significant for an alpha risk of 0.05. Statistical analyzes were performed using Epi Info (v. 3.5.1).

\section{Results}

\section{Population}

In August 2018, 223 patients were included in the cohort and therefore eligible for the present study. Fifteen patients were excluded from the analysis due missing data on genotype and/or initial phenotype (i.e., non neuropsychological). Twenty-eight additional patients were excluded from the analysis due to missing evaluation criteria (Fig. 1).

\section{Characteristics of the whole cohort}

The demographic data of the 180 remaining patients are presented in Table 1. The mean age at the time of the last follow-up visit was 9.6 years. The sex ratio was $1.5(\mathrm{M} / \mathrm{F})$. The main renal findings on ultrasound at the time of diagnosis were: bilateral abnormalities in $96 \%$ of cases, including multicystic kidney dysplasia (16\%), other cysts (47\%), hyperechogenic kidney (68\%), kidney hypoplasia (14\%). Most patients had a normal renal function. Among these 180 patients, $119(66 \%)$ had a $H N F 1 B$ CNV (deletion of the whole gene) and $61(34 \%)$ had an SNV. Special educational need was identified in 16 patients of school age $(9.6 \%)$. All patients of school age in a general educational environment were in the matching patient age grade.

\section{Patients with a HNF1B CNV}

Demographic data of the 119 patients carrying a deletion of the whole gene are presented in Table 1. Ninety-two percent of these patients were of school age.

\section{Patients with HNF1B SNV}

Demographic data of the 61 patients carrying an SNV are presented in Table 1. According to HGVS recommendations, $H N F 1 B$ disease-associated variants were considered to affect or probably affect function in $69.5 \%$ and $30.5 \%$ of cases, respectively. Ninety-two percent of these patients were of school age.

No statistical difference in patient characteristics was found between both subgroups except for urinary tract 


\begin{tabular}{|c|c|c|c|c|c|}
\hline & & $\begin{array}{l}H N F 1 B \\
\text { deletion }\end{array}$ & $\begin{array}{l}H N F 1 B \\
\text { variant }\end{array}$ & Total & $p$-value \\
\hline \multirow{14}{*}{$\begin{array}{l}\text { Demographic } \\
\text { features }\end{array}$} & Gender & & & & \\
\hline & Female & $44(37 \%)$ & $28(46 \%)$ & $72(40 \%)$ & 0.26 \\
\hline & Male & $75(63 \%)$ & $33(54 \%)$ & $108(60 \%)$ & \\
\hline & Mean age (years) & & & & \\
\hline & $\begin{array}{l}\text { At the time of the last follow- } \\
\text { up visit }\end{array}$ & $9.4(4.7)$ & $9.9(5.5)$ & $9.6(5)$ & 0.44 \\
\hline & Ultrasonographic data & & & & \\
\hline & Bilateral anomalies & $114(96 \%)$ & $58(95 \%)$ & $172(96 \%)$ & 1.0 \\
\hline & Multicystic dysplasia & $31(13 \%)$ & $24(21 \%)$ & $55(16 \%)$ & 0.12 \\
\hline & Cysts & $117(49 \%)$ & $53(43 \%)$ & $170(47 \%)$ & 0.31 \\
\hline & Hyperechogenic kidney & $164(68 \%)$ & $81(67 \%)$ & $245(68 \%)$ & 0.63 \\
\hline & Kidney hypoplasia & $26(11 \%)$ & $25(20 \%)$ & $51(14 \%)$ & 0.17 \\
\hline & Renal agenesis & $3(2 \%)$ & $2(2 \%)$ & $5(1 \%)$ & 1.0 \\
\hline & Urinary tract dilation & $13(5 \%)$ & 0 & $13(4 \%)$ & 0.01 \\
\hline & Other & $4(2 \%)$ & 0 & $4(1 \%)$ & 0.30 \\
\hline
\end{tabular}

The percentage of ultrasound features is based on the number of renal units: 360 for the 180 patients analysed, except data missing for three patients (two with a deletion and one with a disease-associated variant). "Cysts" includes cortical, medullary macro, and microcysts. "Urinary tract dilation" includes pyelectasis, dilation secondary to vesicoureteral reflux, hydronephrosis, ureteropelvic junction obstruction, posterior urethral valves. "Other" includes increased kidney size, kidney with poor corticomedullary differentiation

dilation that was more frequent in the group with $H N F 1 B$ deletion.

\section{Prevalence of neuropsychological disorders in patients with a deletion of the whole HNF1B gene}

Among the 119 patients carrying the $H N F 1 B$ deletion $(\mathrm{M} / \mathrm{F}$ ratio 1.7), 110 were of school age, including 96 (87.3\% IC 95\% [81-93]) who had a normal school level, in a general educational environment, as shown in Fig. 1. Special educational needs were identified in 14 patients $(12.7 \%$ [6.5-18.9], M/F ratio 3.6), including medical centers (medical center for cognitive impairment, psychiatric disorder center, and autism center), and special school for learning disabilities. When the analysis was restricted to the subgroup of children older than 6 years old, this proportion was $15.9 \%$ [8.3-23.6]. The proportion of patients with special educational needs is therefore significantly higher for children presenting with $H N F 1 B$ deletion than for French children in general (1.2\%) [14] OR 11.92 [6.8-20.9].

\section{Prevalence of neuropsychological disorders in patients with a HNF1B SNV}

Among the 61 patients carrying the $H N F 1 B$ SNV, 56 were of school age, including 54 (96.4\% [87.8-99.0]) who had a normal school level in a general educational environment as detailed in Fig. 1. Special educational needs (medical center) were identified in two $(3.6 \%$ [1.0-12.1]) patients. The proportion of patients with special educational needs is therefore not significantly different for children presenting with $H N F 1 B$ SNV than for French children in general (1.2\%) [14] OR 3.08 [0.8-12.7].

When comparing neuropsychological outcomes, the proportion of patients with special educational needs was not significantly different between both groups $(p=0.09)$.

\section{Discussion}

The aim of this study was to assess the prevalence of educational difficulties as a proxy for potential neuropsychological disorders in a large pediatric cohort of patients carrying a HNF1B disease-associated variant or deletion. Regarding patients carrying a $H N F 1 B \mathrm{CNV}$ included in this study, the prevalence of patients with severe neuropsychological impairment (i.e., severe enough to disrupt standard schooling) was $12.7 \%$, significantly higher than in general population. This higher risk was not found for patients presenting with a $H N F 1 B$ SNV (3.6\%).

In our cohort, the prevalence of neuropsychiatric disorders within the group of patients carrying a $H N F 1 B$ deletion was less than that reported in previous studies in patients with $H N F 1 B$ or $17 \mathrm{q} 12$ deletions $[5,6,8,9]$. In the first study published on this issue [5], Moreno-De-Luca has reported 18 
patients carrying a $17 \mathrm{q} 12$ microdeletion in the 15,749 cases referred for clinical genetic testing because of autism spectrum disorder, developmental delay and/or intellectual disability, but in none of 4519 controls. Given patient selection criteria in this study, all patients diagnosed with a $17 \mathrm{q} 12$ microdeletion had severe neuropsychiatric disorders so that the authors have suggested that such a microdeletion could "confer a high risk for autism or schizophrenia". Similar conclusions could be drawn from other similar studies $[6,10]$. We assume that our study could have underestimated the prevalence of neuropsychiatric disorders (based on the evaluation criteria used) but, based on our data, the risk of severe neuropsychological disorders affecting schooling seemed to involve a smaller proportion of patients with a $H N F 1 B$ (therefore 17q12) microdeletion than expected, compared with previous studies. Our study, based on a systematic evaluation of patients with a $H N F 1 B$ anomaly diagnosed secondary to kidney abnormalities, could more accurately reflect the actual prevalence of neuropsychiatric disorders in these patients. However, it cannot be excluded that the prevalence of neuropsychiatric disorders could be different in other populations depending on how the disease manifests.

Clissold et al. and Dubois-Laforgue et al. have compared the neurodevelopmental phenotype of patients with diabetes or renal diseases related to $H N F 1 B \mathrm{SNV}$ or CNV $[8,9]$. Thus, their results may be more appropriately compared with our findings. However, the prevalence of neuropsychiatric disorders in patients with $H N F 1 B \mathrm{CNV}$ or SNV was $40 \%$ and $0 \%$, respectively in Clissold study, and $17 \%$ and $11 \%$, respectively in Dubois-Laforgue study while we found a prevalence of $12.7 \%$ and $3.6 \%$, respectively. Even if not similar, our results are therefore closer from those of DuboisLaforgue. Several differences between these two studies and ours could explain this discrepancy. First, patient mean age (15.5 and 46 years, respectively) was higher in the two former studies that also or exclusively included adult patients compared with our study (mean age: 9.6 years). Therefore, it could not be excluded that the prevalence of neuropsychiatric disorders could increase over time in our cohort, specifically for psychiatric disorders such has schizophrenia which develop more frequently during adulthood, after the completion of the schooling period. Second, as patients with diabetes were included in these studies, the prevalence of neurological disorders depending on how the disease manifested could be discussed again. Finally, the main explanation could be the choice of evaluation criteria that differed between these three studies. Indeed, while Clissold has used criteria to detect even mild neuropsychological disorders (that sometimes have no or very little impact on normal schooling), Dubois-Laforgue has used less sensitive evaluation criteria for most included patients (answers provided by referring physicians to questions on intellectual or learning disabilities). We also chose criteria with low sensibility and focus only on the detection of disorders that were severe enough to disrupt normal schooling. Actually, we decided to base our evaluation on such a criterion in order to report data that could have an impact on antenatal counseling. Therefore, the criteria used were far less sensitive than those used in Clissold study to detect neuropsychiatric disorders, but more specific to identify severe neuropsychiatric disorders.

In the present study, even if patients with a HNFIB CNV seem to present more frequently with neuropsychiatric disorder than patients with an SNV, this difference was not statistically significant, probably due to lack of power secondary to low prevalence of neurological impairment as defined in this study.

Although lower than previously reported in some studies, the prevalence of neuropsychiatric disorders in patients carrying a $H N F 1 B$ deletion remains higher than in the general population. Collectively, the data presented here, in addition to those already published [4-6, 8-10, 15], confirm that a neuropsychiatric impairment could be more common in patients with a $H N F 1 B$ (or 17q12) deletion than in general population.

Several pathophysiological hypotheses have been suggested to explain these data. First, variants of other genes encompassed by the 17q12 microdeletion, such as $L H X$ [16] or ACACA [17], could be related to neurological impairments. Second, the neuropsychiatric involvement in patients carrying a 17q12 deletion could be associated with additional chromosomic microdeletions. Interestingly, it has been reported that $10 \%$ of patients carrying a $17 \mathrm{q} 12$ microdeletion may carry a second one [10]. Finally, even if neuropsychiatric disorders could be less common in patients with a $H N F 1 B \mathrm{SNV}$, a direct involvement of the $H N F 1 B$ gene in the neurological phenotype cannot be totally excluded, particularly given its role in transcriptional networks involved in a pathway patterning vertebrate hindbrain [18-20].

Independently of a putative role of the $H N F 1 B$ and/or $17 q 12$ deletion in neuropsychiatric disorders, our study shows that almost $90 \%$ of patients with antenatal features of $H N F 1 B$ abnormalities have a neurobehavioral development normal enough for normal schooling at a mean age of 9.6 years. However, as a few patients with a $17 \mathrm{q} 12$ microdeletion or HNF1B SNV show a severe neuropsychological involvement, several additional hypotheses could be discussed to explain this great interindividual variability. A $17 \mathrm{q} 12$ microdeletion on a single chromosome could not be sufficient to result in a neurological impairment. To develop symptoms, both a deletion and another genetic or chromosomic alteration elsewhere in the genome could be required. Using single-nucleotide polymorphism array analyzes, no other large deletions were found in patients with intellectual disabilities reported by Dubois-Laforgue et al. [9]. Nevertheless, as $10 \%$ of patients carrying a microdeletion actually 
carry at least a second one [10], larger studies are needed to confirm these results. As the $17 \mathrm{q} 12$ microdeletion may either be inherited or occur de novo, it would be of particular interest to study the relationship between the neuropsychological phenotypes of children, those of their parents and the transmission mode of the deletion. Indeed, other phenomena such as parental imprinting or other epigenetic [21] factors could be involved in the development of these neuropsychiatric features. Interestingly, all but one patient in the cohort published by Moreno-De-Luca et al. carried a de novo deletion [5]. Unfortunately, our study did not allow testing this hypothesis because clinical or molecular testing data were not available for the all the parents of the included cases. Another explanation could be based on $H N F 1 B$ variants of the single remaining gene copy (or variants of other remaining genes within the not deleted $17 \mathrm{q} 12$ locus) that are not currently considered as affecting function but could directly or indirectly have an impact on neuropsychiatric development (through, for instance, the amount or function of the residual $H N F 1 B$ protein). These hypotheses deserve to be tested.

To summarize, although carrying $17 \mathrm{q} 12$ deletion significantly increases the risk to show a neuropsychiatric disorder severe enough to impede normal schooling when compared with the general population, most patients with this deletion do not display a severe neuropsychiatric involvement (contrary to the conclusions drawn from more limited studies) [22]. Therefore, the presence of a HNF1B SNV or CNV is not sufficient to predict a severe neurological outcome.

Our study has some limitations. First, this study was not designed to precisely describe the neuropsychological profile of patients carrying a $H N F 1 B \mathrm{CNV}$ or a $17 \mathrm{q} 12$ deletion. Indeed, we used school level as evaluation criterion because it is a reliable data and relevant in the clinical practice. Even if we have tempted to precise as much as possible the neuropsychiatric assessment of the patients with impaired schooling by contacting each clinician, this remains a major limitation of our study as no neuropsychiatric battery examination was conducted. Therefore, mild learning disabilities or behavioral difficulties could not have been detected with the simple evaluation of school level alone. In addition, other factors than genetic variations are known to have an impact on school level (i.e., schooling region, family, ...). Second, despite its wide recruitment, this study only describes the schooling of patients with kidney abnormalities usually diagnosed during pregnancy or early in infancy. Therefore, its conclusion cannot be automatically generalized to the whole population of patients carrying a $H N F 1 B \mathrm{SNV}$ or $\mathrm{CNV}$.

Despite all these limitations, we believe that our study provides solid data that could be used by physicians during patient and parent counseling when an antenatal $H N F 1 B$ molecular abnormality is discussed (either an SNV or a
CNV). Indeed, this study was conducted in the largest pediatric cohort of patients with $H N F 1 B$ SNV and $H N F 1 B /$ $17 q 12$ deletion to date, with a limited number of patients lost to follow-up, and it therefore provides more comprehensive data to assess the impact of these genetic anomalies on patient schooling. Furthermore, since most of our patients were diagnosed secondary to the prenatal identification of ultrasound anomalies and given that most patients with a $H N F 1 B$ SNV or $H N F 1 B / 17 \mathrm{q} 12$ deletion diagnosed prenatally were diagnosed secondary to renal ultrasound anomalies, this study has a low inclusion bias (from an antenatal counseling point of view). Therefore, our findings could certainly be generalized to the population targeted by prenatal counseling.

In conclusion, although these data may not be generalized to the whole population of patients carrying a $H N F 1 B$ SNV or a $H N F 1 B / 17 \mathrm{q} 12$ deletion, our results showing that almost $90 \%$ of patients with kidney abnormalities leading to the diagnosis of $H N F 1 B / 17 \mathrm{q} 12$ deletion or SNV had a normal school level in a general educational environment, should be put in balance with the increased risk of neuropsychiatric involvement in some of these patients. These data need to be taken into account for antenatal counseling.

Funding This work was funded by grants from the following patient associations: AdéTIRE, AIRG, AFD, and Océane pour la vie des Reins.

\section{Compliance with ethical standards}

Conflict of interest The authors declare that they have no conflict of interest.

Publisher's note: Springer Nature remains neutral with regard to jurisdictional claims in published maps and institutional affiliations.

\section{References}

1. Barbacci E. Variant hepatocyte nuclear factor 1 is required for visceral endoderm specification. Development. 1999; 126AD:4795-805. 11

2. El-Khairi R, Vallier L. The role of hepatocyte nuclear factor $1 \beta$ in disease and development. Diabetes Obes Metab. 2016;18:23-32.

3. Heidet L, Decramer S, Pawtowski A, Moriniere V, Bandin F, Knebelmann B, et al. Spectrum of HNF1B mutations in a large cohort of patients who harbor renal diseases. Clin J Am Soc Nephrol. 2010;5:1079-90.

4. Laffargue F, Bourthoumieu S, Llanas B, Baudouin V, Lahoche A, Morin D, et al. Towards a new point of view on the phenotype of patients with a $17 \mathrm{q} 12$ microdeletion syndrome. Arch Dis Child. 2015;100:259-64.

5. Moreno-De-Luca D, Mulle JG, Kaminsky EB, Sanders SJ, Myers SM, Adam MP, et al. Deletion $17 \mathrm{q} 12$ is a recurrent copy number variant that confers high risk of autism and schizophrenia. Am J Hum Genet. 2010;87:618-30.

6. Rasmussen M, Vestergaard EM, Graakjaer J, Petkov Y, Bache I, Fagerberg $\mathrm{C}$, et al. 17q12 deletion and duplication syndrome in 
Denmark - a clinical cohort of 38 patients and review of the literature. Am J Med Genet Part A. 2016 http://doi.wiley.com/10. 1002/ajmg.a.37848. Accessed 23 Aug 2016.

7. Chen C-P, Chang S-D, Wang T-H, Wang L-K, Tsai J-D, Liu Y$\mathrm{P}$, et al. Detection of recurrent transmission of $17 \mathrm{q} 12$ microdeletion by array comparative genomic hybridization in a fetus with prenatally diagnosed hydronephrosis, hydroureter, and multicystic kidney, and variable clinical spectrum in the family. Taiwan J Obstet Gynecol. 2013;52:551-7.

8. Clissold RL, Shaw-Smith C, Turnpenny P, Bunce B, Bockenhauer D, Kerecuk L, et al. Chromosome 17q12 microdeletions but not intragenic HNF1B mutations link developmental kidney disease and psychiatric disorder. Kidney Int. 2016 http://www. sciencedirect.com/science/article/pii/S0085253816301156. Accessed 23 Aug 2016.

9. Dubois-Laforgue D, Bellanné-Chantelot C, Charles P, Jacquette A, Larger E, Ciangura C, et al. Intellectual disability in patients with MODY due to hepatocyte nuclear factor 1B (HNF1B) molecular defects. Diabetes Metab. 2017;43:89-92.

10. Girirajan S, Rosenfeld JA, Coe BP, Parikh S, Friedman N, Goldstein A, et al. Phenotypic heterogeneity of genomic disorders and rare copy-number variants. New Engl J Med. 2012;367:1321-31.

11. Decramer S, Parant O, Beaufils S, Clauin S, Guillou C, Kessler S, et al. Anomalies of the TCF2 gene are the main cause of fetal bilateral hyperechogenic kidneys. J Am Soc Nephrol. 2007;18:923-33.

12. Bellanne-Chantelot C, Clauin S, Chauveau D, Collin P, Daumont $\mathrm{M}$, Douillard $\mathrm{C}$, et al. Large genomic rearrangements in the hepatocyte nuclear factor-1 (TCF2) gene are the most frequent cause of maturity-onset diabetes of the young type 5. Diabetes. 2005;54:3126-32.

13. Sequence Variant Nomenclature. https://varnomen.hgvs.org/bgmaterial/basics/. Accessed 19 Jun 2019.
14. Rosenwald $\mathrm{F}$. Repères et références statistiques sur les enseignements, la formation et la recherche 2018. education.gouv.fr: 2018. http://www.education.gouv.fr/cid57096/reperes-et-references-sta tistiques.html. Accessed 23 Dec 2018.

15. Loirat C, Bellanne-Chantelot C, Husson I, Deschenes G, Guigonis $\mathrm{V}$, Chabane N. Autism in three patients with cystic or hyperechogenic kidneys and chromosome 17q12 deletion. Nephrol Dial Transpl. 2010;25:3430-3.

16. Abellán A, Vernier B, Rétaux S, Medina L. Similarities and differences in the forebrain expression of Lhx 1 and Lhx 5 between chicken and mouse: Insights for understanding telencephalic development and evolution. J Comp Neurol. 2010;518:3512-28.

17. Girirajan S, Dennis MY, Baker C, Malig M, Coe BP, Campbell $\mathrm{CD}$, et al. Refinement and discovery of new hotspots of copynumber variation associated with autism spectrum disorder. Am J Hum Genet. 2013;92:221-37.

18. Bae C-J, Jeong J, Saint-Jeannet J-P. A novel function for Egr4 in posterior hindbrain development. Sci Rep. 2015;5:7750.

19. Wiellette EL. vhnf1 and Fgf signals synergize to specify rhombomere identity in the zebrafish hindbrain. Development. 2003;130:3821-9.

20. Makki N, Capecchi MR. Identification of novel Hoxa1 downstream targets regulating hindbrain, neural crest and inner ear development. Dev Biol. 2011;357:295-304.

21. Clissold RL, Ashfield B, Burrage J, Hannon E, Bingham C, Mill J, et al. Genome-wide methylomic analysis in individuals with HNF1B intragenic mutation and $17 \mathrm{q} 12$ microdeletion. Clin Epigenetics. 2018;10. https://clinicalepigeneticsjournal.biomedcentral.com/a rticles/10.1186/s13148-018-0530-z. Accessed 26 Dec 2018.

22. Gilboa Y, Perlman S, Pode-Shakked N, Pode-Shakked B, Shrim A, Azaria-Lahav E, et al. Prenatal diagnosis of 17q12 deletion syndrome: from fetal hyperechogenic kidneys to high risk for autism: prenatal echogenic kidneys and autism in 17q12 deletions. Prenat Diagn 2016;36:1027-32.

\section{Affiliations}

Fanny Laliève ${ }^{1} \cdot$ Stéphane Decramer ${ }^{2} \cdot$ Laurence Heidet $^{3} \cdot$ Véronique Baudouin $^{4} \cdot$ Annie Lahoche $^{5} \cdot$ Brigitte Llanas $^{6}$. Pierre Cochat $^{7}$ - Julie Tenenbaum ${ }^{8}$ - Marie-Pierre Lavocat ${ }^{9}$ - Philippe Eckart ${ }^{10}$ - Françoise Broux $^{11}$. Gwenaelle Roussey $^{12} \cdot$ Sylvie Cloarec $^{13} \cdot$ Isabelle Vrillon $^{14} \cdot$ Olivier Dunand $^{15} \cdot$ Lucie Bessenay $^{16}$. Michel Tsimaratos ${ }^{17}$. François Nobili ${ }^{18}$. Christine Pietrement ${ }^{19}$ • Loïc De Parscau ${ }^{20}$ • Valérie Bonneville ${ }^{21}$. Nicolas Rodier ${ }^{22}$ - Cécile Saint-Martin ${ }^{23}$. Nicolas Chassaing ${ }^{24}$ - Laurence Michel-Calemard ${ }^{25}$ - Vincent Moriniere ${ }^{26}$. Christine Bellanné-Chantelot ${ }^{23}$ - Claire Bahans ${ }^{1} \cdot$ Vincent Guigonis $\mathbb{C}^{1,22,27}$

1 Service de Pédiatrie, CHU de Limoges, Limoges, France

2 Service de Néphrologie Pédiatrique, CHU de Toulouse, Toulouse, France

3 Service de Néphrologie Pédiatrique, Hôpital Necker, APHP, Paris, France

4 Service de Néphrologie Pédiatrique, Hôpital Robert Debré, APHP, Paris, France

5 Service de Néphrologie Pédiatrique, CHU Jeanne de Flandres, Lille, France

6 Service de Néphrologie Pédiatrique, CHU de Bordeaux, Bordeaux, France

7 Service de Néphrologie Pédiatrique, Hôpital Femme Mère Enfant, Lyon, France
8 Service de Néphrologie Pédiatrique, CHU de Montpellier, Montpellier, France

9 Service de Néphrologie Pédiatrique, CHU de Saint-Etienne, SaintEtienne, France

10 Service de Néphrologie Pédiatrique, CHU de Caen, Caen, France

11 Service de Néphrologie Pédiatrique, CHU de Rouen, Rouen, France

12 Service de Néphrologie Pédiatrique, CHU de Nantes, Nantes, France

13 Service de Néphrologie Pédiatrique, CHU de Tours, Tours, France

14 Service de Néphrologie Pédiatrique, CHU de Nancy, Nancy, France 
15 Service de Néphrologie Pédiatrique, CHU de la Réunion, SaintDenis, France

16 Service de Néphrologie Pédiatrique, CHU de Clermont, ClermontFerrand, France

17 Service de Néphrologie Pédiatrique, CHU de Marseille, Marseille, France

18 Service de Néphrologie Pédiatrique, CHU de Besançon, Besançon, France

19 Service de Néphrologie Pédiatrique, CHU de Reims, Reims, France

20 Service de Pédiatrie, CHU de Brest, Brest, France

21 Service de Pédiatrie, $\mathrm{CH}$ de Chollet, Chollet, France
22 CIC 1435, CHU de Limoges, Limoges, France

23 Département de génétique, Assistance Publique-Hôpitaux de Paris (AP-HP) Hôpitaux Universitaires Pitié Salpêtrière-Charles Foix, Sorbonne Université, Paris, France

24 CHU Toulouse, Service de Génétique Médicale, Hôpital Purpan, Toulouse, France

25 Service de Biochimie Biologie Moléculaire Grand Est; UM Pathologies Endocriniennes, Rénales, Musculaires et Mucoviscidose; LBMMS, Hospices Civils de Lyon, Bron, France

26 Service de Génétique, Hôpital Necker, APHP, Paris, France

27 UMR CNRS 7276, Limoges, France 